\title{
Circular RNA Expression Profile of Pancreatic Ductal Adenocarcinoma Revealed by Microarray
}

\author{
Haimin $\mathrm{Li}^{\mathrm{a}}$ Xiaokun Hao ${ }^{\mathrm{a}, \mathrm{b}}$ Huimin Wang ${ }^{\mathrm{a}}$ Zhengcai Liu ${ }^{\mathrm{a}}$ Yong He $\mathrm{He}^{\mathrm{a}}$ Meng Pu \\ Hongtao Zhang ${ }^{a}$ Hengchao Yu ${ }^{a}$ Juanli Duan ${ }^{a}$ Shibin $\mathrm{Qu}^{\mathrm{a}}$ \\ aDepartment of Hepatobiliary Surgery, Xijing Hospital, Fourth Military Medical University, ${ }^{\text {bDepartment }}$ \\ of General Surgery, 141 Hospital, Xi'an, China
}

\section{Key Words}

Circular RNA • Noncoding RNA • Pancreatic cancer • Microarray

\begin{abstract}
Background/Aims: Circular RNAs (circRNAs) are a special novel type of a stable, diverse and conserved noncoding RNA in mammalian cells. Particularly in cancer, circRNAs have been reported to be widely involved in the physiological/pathological process of life. However, it is unclear whether circRNAs are specifically involved in pancreatic ductal adenocarcinoma (PDAC).

Methods: We investigated the expression profile of circRNAs in six PDAC cancer samples and paired adjacent normal tissues using microarray. A high-throughput circRNA microarray was used to identify dysregulated circular RNAs in six PDAC patients. Bioinformatic analyses were applied to study these differentially expressed circRNAs. Furthermore, quantitative reverse transcription polymerase chain reaction (qRT-PCR) was performed to confirm these results. Results: We revealed and confirmed that a number of circRNAs were dysregulated, which suggests a potential role in pancreatic cancer. Conclusions: this study demonstrates that clusters of circRNAs are aberrantly expressed in PDAC compared with normal samples and provides new potential targets for the future treatment of PDAC and novel insights into PDAC biology.

\section{Introduction}

Pancreatic cancer remains one of the most common and deadly cancers. Pancreatic ductal adenocarcinoma (PDAC) accounts for $>85 \%$ of pancreatic cancer $[1,2]$. The incidence of PDAC has been increasing in the last 20 years. However, the five-year survival rate of PDAC is still at approximately $5 \%[3,4]$. PDAC has a poor prognosis because of its high malignancy and tardive symptoms, which make its early diagnosis difficult. The treatments of PDAC Haimin Li, Xiaokun Hao and Huimin Wang contributed equally to this manuscript. 
primarily depend on surgery. Moreover, because PDAC is often diagnosed at an advanced stage, fewer than $20 \%$ of the patients with PDAC have the resectable opportunity, and the postoperative average 5-year survival rate is only $15 \%$ to $20 \%$. Many of these patients die from relapse or metastasis [5].

The majority of human transcriptome was proven to be noncoding RNAs. Only less than $2 \%$ of the human genome encodes proteins [6]. Accumulating evidence has shown that noncoding RNAs, such as microRNA (miRNA) and long noncoding RNA (lncRNA), play a key regulatory role in the cellular physiological process and the cancer biological process $[7,8]$. Circular RNA has recently been identified as one novel type of endogenous noncoding RNA. Unlike linear RNAs terminated with 5' caps and 3' tails, circular RNAs form covalently closed loop structures with neither $5^{\prime}$ to $3^{\prime}$ polarity nor polyadenylated tail [9]. Thousands of stable, conserved circRNAs often show tissue/developmental stage-specific expression [10-13]. More importantly, recent research has demonstrated that circRNAs are widely involved in the physiological/pathological process, such as neuronal differentiation, synaptogenesis, neurological disorders, angiogenesis, prion disease or cancer [11, 14-19]. Likewise, circRNAs were described as a class of aging biomarkers in Drosophila [20] and as putative disease biomarkers in human saliva [21]. Circular ANRIL (CANRIL) was reported to regulate INK4/ARF expression and correlate with atherosclerotic vascular disease risk [22]. $\mathrm{Li}$ and others have also found that hsa_circ_002059, a typical circular RNA, was significantly down-regulated in gastric cancer (GC) tissues compared with paired adjacent nontumorous tissues, suggesting that it may be a potential novel and stable biomarker for the diagnosis of gastric carcinoma [23]. These findings indicated that circRNAs may play a crucial role in the process of life. However, few papers have reported the relationship between circRNAs and cancer. Whether circRNAs expression is dysregulated in PDAC is unknown to us. The biological functions of circRNAs in PDAC remain to be clarified.

In this study, we explored the circRNAs expression profile in six pairs of PDAC samples compared with matched adjacent normal tissues. Several differentially expressed circRNAs were evaluated by quantitative reverse transcription polymerase chain reaction (qRT-PCR) in 20 pairs of tissues. Our results showed that circRNAs expression profiles are significantly different between normal pancreas tissues and PDAC. This finding suggests that differentially expressed circRNAs may be involved in the initiation and process of PDAC and may provide molecular biomarkers or therapeutic targets for PDAC.

\section{Materials and Methods}

Patient samples

This study was approved by the institutional Ethics Review Board of Xijing Hospital of the Fourth Military Medical University and was conducted according to the Ethical Guidelines for Human Genome/ Gene Research issued by the Chinese Government. The PDAC samples and corresponding normal tissues were prospectively collected from 20 patients of Xijing Hospital from July 2013 to January 2015. Of these 20 patients, six patients (for basic clinical data, see Table 1) were also used for the microarray analysis of circRNA. All of the patients underwent neither chemotherapy nor radiotherapy before operation. The diagnosis of PDAC was confirmed by histopathology. Tumor stages were determined according to the American Joint Committee on Cancer (AJCC) tumor-node-metastasis (TNM) staging criteria. Each sample was snap-frozen in liquid nitrogen immediately after resection and subsequently was stored at $-130^{\circ} \mathrm{C}$ until use.

RNA extraction and quality control

Total RNA was isolated from six PDAC and paired adjacent normal tissues using TRIzol reagent (Invitrogen, Carlsbad, CA, USA), according to the manufacturer's protocol. RNA integrity was assessed using standard denaturing agarose gel electrophoresis. The total RNA from each specimen was quantified and quality assurance was provided using NanoDrop ND-1000 (NanoDrop, Wilmington, DE, USA). 


\begin{tabular}{|c|c|c|}
\hline Co & Cell Physiol Biochem 2016;4 & $0: 1334-1344$ \\
\hline ind Biochemistry & $\begin{array}{l}\text { DOI: 10.1159/000453186 } \\
\text { Published online: December 19, } 2016\end{array}$ & $\begin{array}{l}\text { O } 2016 \text { The Author(s). Published by S. Karger AG, Basel } \\
\text { www.karger.com/cpb }\end{array}$ \\
\hline
\end{tabular}

CircRNA microarray and Table 1. Clinical characteristics of the patients computational analysis

Human 8x15K circRNA Array is manufactured by Arraystar Technologies (Rockville, MD, USA). A total of 5396 circRNAs were collected from three published papers $[11,13,24]$. Each circRNA

\begin{tabular}{lllll}
\hline Patient No. & Age & Gender & $\begin{array}{l}\text { Histologic } \\
\text { differentiation }\end{array}$ & TNM Stage \\
\hline 1 & 45 & Male & Moderate-poorly & T3N1M0 \\
2 & 42 & Female & Well & T3N1M0 \\
3 & 55 & Female & Well-moderately & T3N1M0 \\
4 & 61 & Female & Moderately & T2N0M0 \\
5 & 61 & Male & Moderately & T2N0M0 \\
6 & 74 & Female & Poorly & T2N0M0 \\
\hline
\end{tabular}
was accurately identified using a specific probe targeting the circRNA-specific junction. Sample labeling, array hybridization, raw data extraction and quality control were performed based on the previous study (Fig. 1) [25]. Quantile normalization of raw data and subsequent data processing were performed using the $\mathrm{R}$ software package ( $\mathrm{R}$ version 3.1.2).

The scatter plot is a visualization method used for assessing the circRNA expression variation. Differentially expressed circRNAs with statistical significance (fold changes $\geq 1.5$ and $P<0.05$ ) between groups were identified using fold change cut-off or volcano plot filtering, respectively. Gene ontology (GO) analysis and pathway analysis for circRNAs gene symbols were applied to determine the roles that these differentially expressed circRNAs played in GO terms of biological pathways. The circRNA/microRNA interaction was predicted using Arraystar's home-made miRNA target prediction software based on TargetScan and miRanda. All of the differentially expressed circRNAs were annotated in detail according to the circRNA/miRNA interaction information. The microarray work was performed by KangChen Bio-tech, Shanghai, People's Republic of China.

\section{qRT-PCR validation assay}

Total RNA was extracted from 20 pairs of PDAC and normal tissues using TRIzol reagent according to standard procedures. The total RNAs were reverse-transcribed into cDNA with random primer using Transcriptor First Strand cDNA Synthesis Kit (Roche, Penzberg, Germany) according to the manufacturer's instructions. Seven differentially expressed circRNAs were measured by qRT-PCR using FastStart Essential DNA Green Master (Roche) on Bio-Rad iQ5 instrument (Bio-Rad, USA). The reaction conditions were as follows: $95^{\circ} \mathrm{C}$ for $10 \mathrm{~min}$ and 40 cycles of $95^{\circ} \mathrm{C}$ for $10 \mathrm{~s}, 60^{\circ} \mathrm{C}$ for $15 \mathrm{~s}$ and $72{ }^{\circ} \mathrm{C}$ for $20 \mathrm{~s}$. The RNA levels were normalized to human $\beta$-actin. All of the quantitative PCR reactions were conducted in triplicate. Divergent primers, rather than the more commonly used convergent primers, were designed for circRNAs (Fig. 2). We

Fig. 1. Experiment workflow of microarray expression profile of circRNAs.

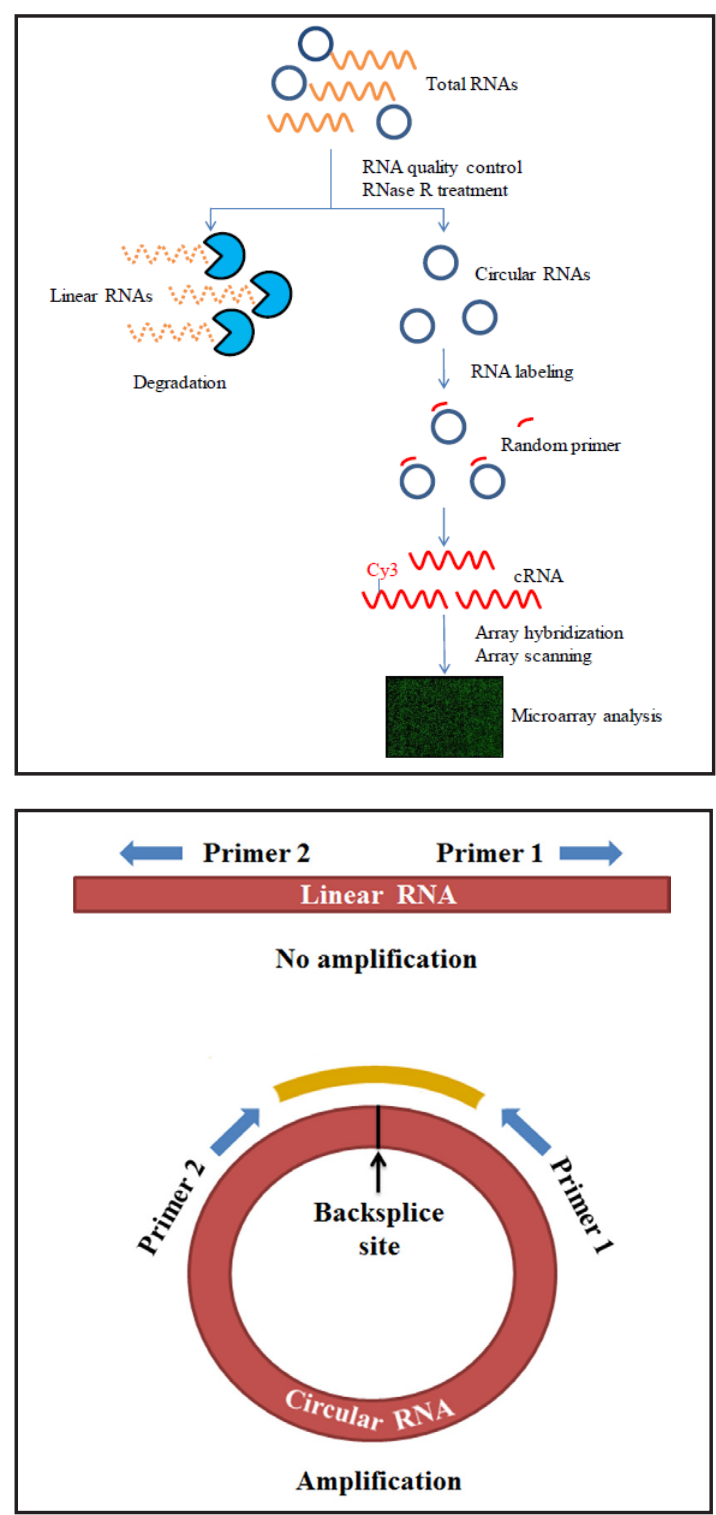

Fig. 2. Schematic diagram of the PCR primers used to specifically detect circular transcripts. Divergent PCR primers were designed on linear cDNA thereby leading to amplification impossible. However, these primers are convergent rendering a circRNA-specific amplicon (yellow pieces) on cDNA derived from circRNA. 
verified the specificity of the PCR appearance of a single-peak in the melt-curve suggested that the specificity of the PCR products. All of the primers are listed in Table 2 .

\section{Statistical analyses}

A statistical analysis was performed using Student's $t$-test to compare two variables of the microarray data. The differences with fold change (FC) $\geq 1.5$ and $P<0.05$ were considered to be statistically significant on microarray analysis. The false discovery rate (FDR) was calculated to correct the $P$ value on microarray analysis. The expression level of each circRNA was represented as fold change using the $2^{-\Delta \Delta \mathrm{Ct}}$ method on qRTPCR analysis.

\section{Results}

\section{Overview of circRNA profiles}

To study the expression profile of circRNAs in PDAC, we performed the circRNA expression profiles in human PDAC and matched normal tissues using the microarray analysis. The box plot is a convenient way to quickly visualize the distributions of a dataset for the circRNAs profiles. After normalization, the distributions of $\log 2$ ratios among twelve samples are nearly the same (Fig. 3A). The scatter plot of circRNA expression profile was used to assess the variations between the two groups (Fig. 3B). Differentially expressed primers using the BLAST. The

Table 2. Primers used for qRT-PCR analysis of circRNA and mRNA levels. F: Forward; R: Reverse; PS: Product size

\begin{tabular}{lll}
\hline Target ID & Primer sequence 5'-3' & PS (bp) \\
\hline hsa_circ_0001946 & F: ACGTCTCCAGTGTGCTGA & 83 \\
& R: CTTGACACAGGTGCCATC & \\
hsa_circ_0005397 & F:GTCATCTGTATAGTGTATGCCGTTA & 138 \\
& $\begin{array}{l}\text { R: CAGCTGGAATGGTGATTTCTT } \\
\text { hsa_circ_0006913 }\end{array}$ & $\begin{array}{l}\text { F: AAACCCAAGAAACTGCTGTCG } \\
\text { R: CTGGAAATCCCTGATGTGCTC }\end{array}$ \\
hsa_circ_0000257 & F: AGGATGAGCCACCAAAGAAC & 138 \\
& R: TGTCTAAGAGGGAAGAGGCATT & 118 \\
hsa_circ_0005785 & F: CTCGCACCTTGTCGCTTAGAT \\
& $\begin{array}{l}\text { R: TCACAGCATTCCGATATTCCTT } \\
\text { hsa_circ_0041150 }\end{array}$ & F: GGAAGAAAGTCTGCACCAAATG \\
& R: TTGGGGCAAACCCACTGAT & 120 \\
hsa_circ_0008719 & F:CCCCCTTAAACAACTTCTCCG & 178 \\
& R: GCGTGGTACGCTGTCACCTA & \\
B-actin & F: CATGTACGTTGCTATCCAGGC & 216 \\
& R: CTCCTTAATGTCACGCACGAT & 250 \\
\hline
\end{tabular}

Table 3. Top 40 differently expressed circRNAs in pancreatic cancer. FDR, false discover rate; FC, fold change

\begin{tabular}{|c|c|c|c|c|c|c|c|c|}
\hline circRNA & P-value & FDR & $\mathrm{FC}$ & Regulation & circRNA_type & chrom & strand & Gene Symbol \\
\hline hsa_circ_0072088 & 0.002674 & 0.071798 & 8.703006 & up & exonic & $\operatorname{chr} 5$ & - & ZFR \\
\hline hsa_circ_0030235 & 0.00166 & 0.071798 & 7.989893 & up & exonic & chr13 & - & RCBTB2 \\
\hline hsa_circ_0001946 & 0.047146 & 0.156034 & 7.07192 & up & antisense & $\operatorname{chr} \mathrm{X}$ & + & CDR1 \\
\hline hsa_circ_0060055 & 0.001865 & 0.071798 & 6.908848 & up & exonic & chr20 & - & EIF6 \\
\hline hsa_circ_0005397 & 0.00298 & 0.071798 & 5.417164 & up & exonic & chr17 & + & RHOT1 \\
\hline hsa_circ_0007895 & 0.003081 & 0.071798 & 4.785208 & up & exonic & chr1 1 & - & EYA3 \\
\hline hsa_circ_0000520 & 0.007873 & 0.078323 & 4.741711 & up & intragenic & chr14 & & RPPH1 \\
\hline hsa_circ_0000326 & 0.028084 & 0.124181 & 4.633038 & up & intragenic & chr11 & + & MALAT1 \\
\hline hsa_circ_0008514 & 0.004973 & 0.073501 & 4.588874 & up & exonic & chr6 & & RTN4IP1 \\
\hline hsa_circ_0092337 & 0.026211 & 0.120842 & 4.515606 & up & intronic & chr16 & + & RPL13 \\
\hline hsa_circ_0011385 & 0.004817 & 0.073095 & 4.446248 & up & exonic & chr1 & + & EIF3I \\
\hline hsa_circ_0018004 & 0.000584 & 0.070223 & 4.338299 & up & exonic & chr10 & + & PDSS1 \\
\hline hsa_circ_0092314 & 0.031995 & 0.131431 & 4.255017 & up & intronic & chr22 & + & RANBP1 \\
\hline hsa_circ_0070934 & 0.003034 & 0.071798 & 4.159216 & up & exonic & chr4 & + & LARP1B \\
\hline hsa_circ_0066147 & 0.002262 & 0.071798 & 3.958722 & up & exonic & chr3 & - & SFMBT1 \\
\hline hsa_circ_0083756 & 0.020032 & 0.107925 & 3.901264 & up & exonic & chr8 & - & TRIM35 \\
\hline hsa_circ_0003763 & 0.007687 & 0.078126 & 3.821682 & up & exonic & chr3 & - & GSK3B \\
\hline hsa_circ_0009065 & 0.000935 & 0.071798 & 3.794776 & up & exonic & chr16 & + & BFAR \\
\hline hsa_circ_0062389 & 0.022441 & 0.112868 & 3.610354 & up & exonic & chr22 & - & PI4KA \\
\hline hsa_circ_0054970 & 0.008135 & 0.078323 & 3.492835 & up & exonic & chr2 & + & SLC1A4 \\
\hline hsa_circ_0013587 & 0.017068 & 0.10116 & 4.51013 & down & exonic & chr1 & + & LRIG2 \\
\hline hsa_circ_0075410 & 0.006511 & 0.076306 & 3.850366 & down & exonic & chr6 & + & DUSP22 \\
\hline hsa_circ_0008768 & 0.023718 & 0.116291 & 3.30527 & down & exonic & chr2 & + & ASAP2 \\
\hline hsa_circ_0080712 & 0.003582 & 0.071798 & 3.102067 & down & exonic & $\operatorname{chr} 7$ & - & HIP1 \\
\hline hsa_circ_0000257 & 0.002671 & 0.071798 & 3.099689 & down & exonic & chr10 & + & NOLC1 \\
\hline hsa_circ_0002733 & 0.001106 & 0.071798 & 3.088806 & down & exonic & chr1 & + & PLEKHM2 \\
\hline hsa_circ_0079385 & 0.043655 & 0.150063 & 3.035551 & down & exonic & chr7 & + & ZDHHC4 \\
\hline hsa_circ_0092367 & 0.045324 & 0.153226 & 2.980006 & down & intronic & chr15 & + & SNURF-SNRPN \\
\hline hsa_circ_0016404 & 0.008468 & 0.078377 & 2.751197 & down & exonic & chr1 & + & TATDN3 3 \\
\hline hsa_circ_0006502 & 0.009531 & 0.082726 & 2.691025 & down & exonic & chr9 & - & WDR85 \\
\hline hsa_circ_0041150 & 0.005432 & 0.073907 & 2.658452 & down & exonic & chr17 & - & RPH3AL \\
\hline hsa_circ_0026652 & 0.021238 & 0.110203 & 2.620033 & down & exonic & chr12 & + & PCBP2 \\
\hline hsa_circ_0052455 & 0.036175 & 0.138672 & 2.609352 & down & exonic & chr2 & - & MYT1L \\
\hline hsa_circ_0006913 & 0.004749 & 0.073095 & 2.544848 & down & exonic & chr1 & + & ARID1A \\
\hline hsa_circ_0072279 & 0.010106 & 0.084843 & 2.516163 & down & exonic & chr5 & & NUP155 \\
\hline hsa_circ_0003218 & 0.023466 & 0.11547 & 2.505041 & down & exonic & chr2 & + & BMPR2 \\
\hline hsa_circ_0002818 & 0.009458 & 0.082388 & 2.488319 & down & exonic & $\operatorname{chr} \mathrm{X}$ & - & DHRSX \\
\hline hsa_circ_0027774 & 0.008569 & 0.078953 & 2.451236 & down & exonic & chr12 & + & METAP2 \\
\hline hsa_circ_0029636 & 0.022359 & 0.112592 & 2.429831 & down & exonic & chr13 & + & ZMYM2 \\
\hline hsa_circ_0002191 & 0.039454 & 0.142531 & 2.428666 & down & exonic & chr9 & + & C9orf3 \\
\hline
\end{tabular}
circRNAs with statistical significance between the two groups were identified using volcano plot filtering (Fig. 3C). The microarray data had been deposited in the National Center for Biotechnology Information (NCBI) Gene Expression Omnibus (GEO). The GEO accession number is GSE69362 (www.ncbi.nlm.nih.gov/geo/query/acc.cgi?acc=GSE69362). We found that 351 circRNAs were differentially expressed $(\mathrm{FC} \geq 1.5$ and $P<0.05$ ) between PDAC and the 

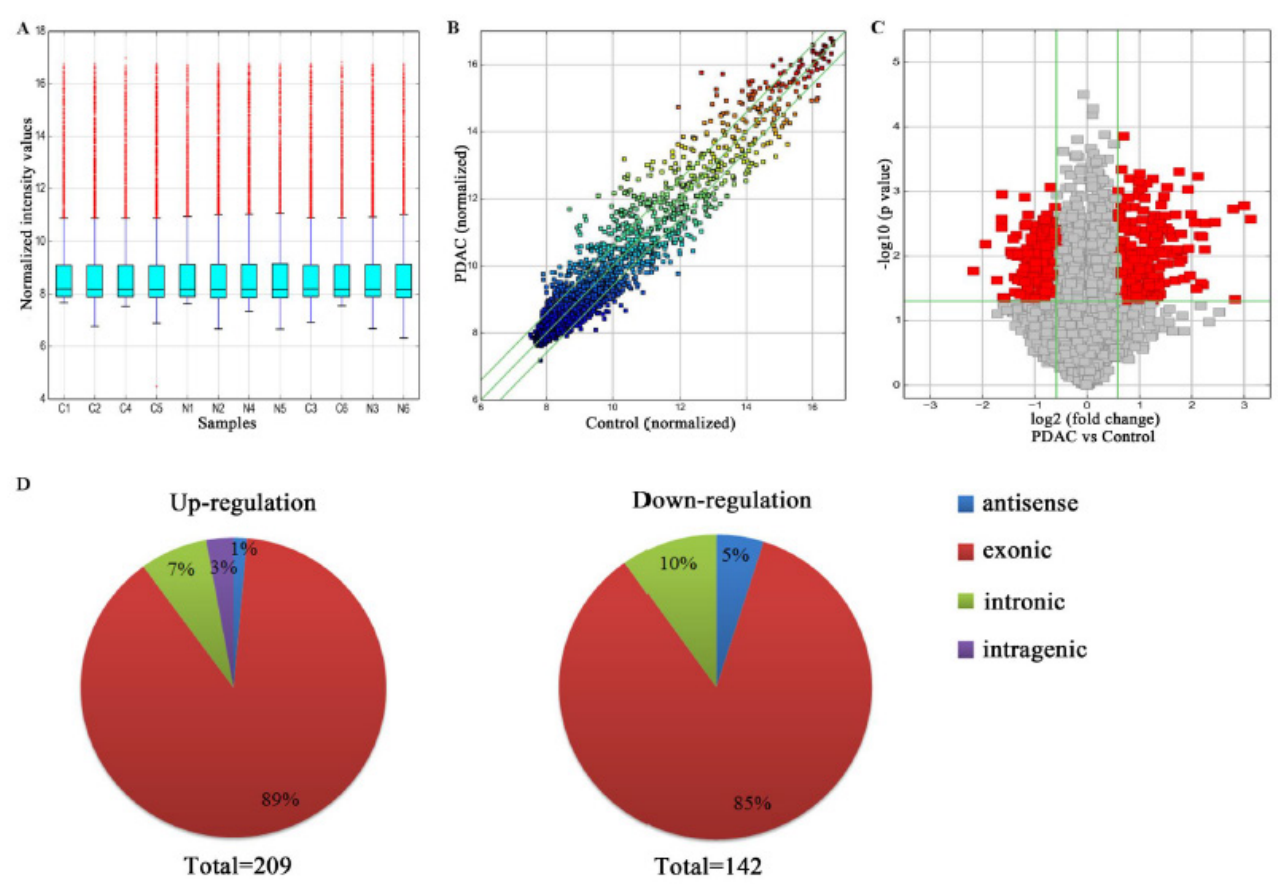

Fig. 3. Overview of the microarray signatures. (A) The box plot is a convenient way to quickly visualize the distributions of a dataset for the circRNAs profiles. After normalization, the distributions of log2 ratios among twelve samples are nearly the same (C: cancer tissue; N: paired adjacent normal tissue). (B) The scatter plot shows the circRNA expression variation between the PDAC and matched adjacent normal samples. The values of $X$ and $Y$ axes in the scatter plot are the averaged normalized signal values of groups of samples (log2 scaled). The green lines are fold change lines. The circRNAs above the top green line and below the bottom green line indicated more than 1.5-fold change of circRNAs between the two groups of samples. (C) Volcano plot of the differentially expressed circRNAs. The vertical lines correspond to 1.5-fold up and down, respectively, and the horizontal line represents $P=0.05$. The red point in the plot represents the differentially expressed circRNAs with statistical significance. (D) Classification of dysregulated circRNAs.

paired normal tissues, of which top 40 differently expressed circRNAs were listed in Table 3. Among these, 209 circRNAs were up-regulated and 142 circRNAs were down-regulated in tumor samples. We summarized the categary of dysregulated circRNAs (Fig. 3D). Among the up-regulated circRNAs, there are 3 antisense, 185 exonic, 15 intronic and 6 intragenic. Among the down-regulated circRNAs, there are 7 antisense, 121 exonic and 14 intronic.

\section{Validation of circRNA expression}

To verify the microarray data, we randomly selected seven differentially expressed circRNAs, including two up-regulated circRNAs and five down-regulated circRNAs (including hsa_circ_0001946, hsa_circ_0005397, hsa_circ_0006913, hsa_circ_0000257, hsa_circ_0005785, hsa_circ_0041150, and hsa_circ_0008719). We validated their expression levels via qRT-PCR in 20 sets of PDAC tissues and adjacent normal tissues. We found that the expression patterns of seven circRNAs were consistent with the microarray data (Fig. 4).

\section{CircRNAs gene symbols GO analysis and pathway analysis}

Recent research has revealed that circRNAs are primarily generated from exons or introns of their parental genes $[9,11,12]$ and can regulate the expression of parental genes $[19,24,26]$. To evaluate the circRNA attributes in the biological process, cellular components and molecular functions, and in pathways, we conducted GO analysis and pathway analysis for circRNAs gene symbols to speculate circRNA potential functions. The lower the $P$ value, the more significant the correlation ( $P$ value $<0.05$ is recommended). We found that the most 
Fig. 4. Comparison of circRNAs expression levels between microarray and qRT-PCR results. Two up-regulated and five down-regulated differentially expressed circRNAs were validated by qRT-PCR in 20 PDAC tissues compared with paired adjacent normal tissues. The Y-axis of the columns in the chart represents the log2-transformed median fold changes $(\mathrm{C} / \mathrm{N})$ in expression across the 20 patients of each of the seven circRNAs validated. The qRT-PCR results are consistent with the microarray data, and presented as the mean and standard deviation values.
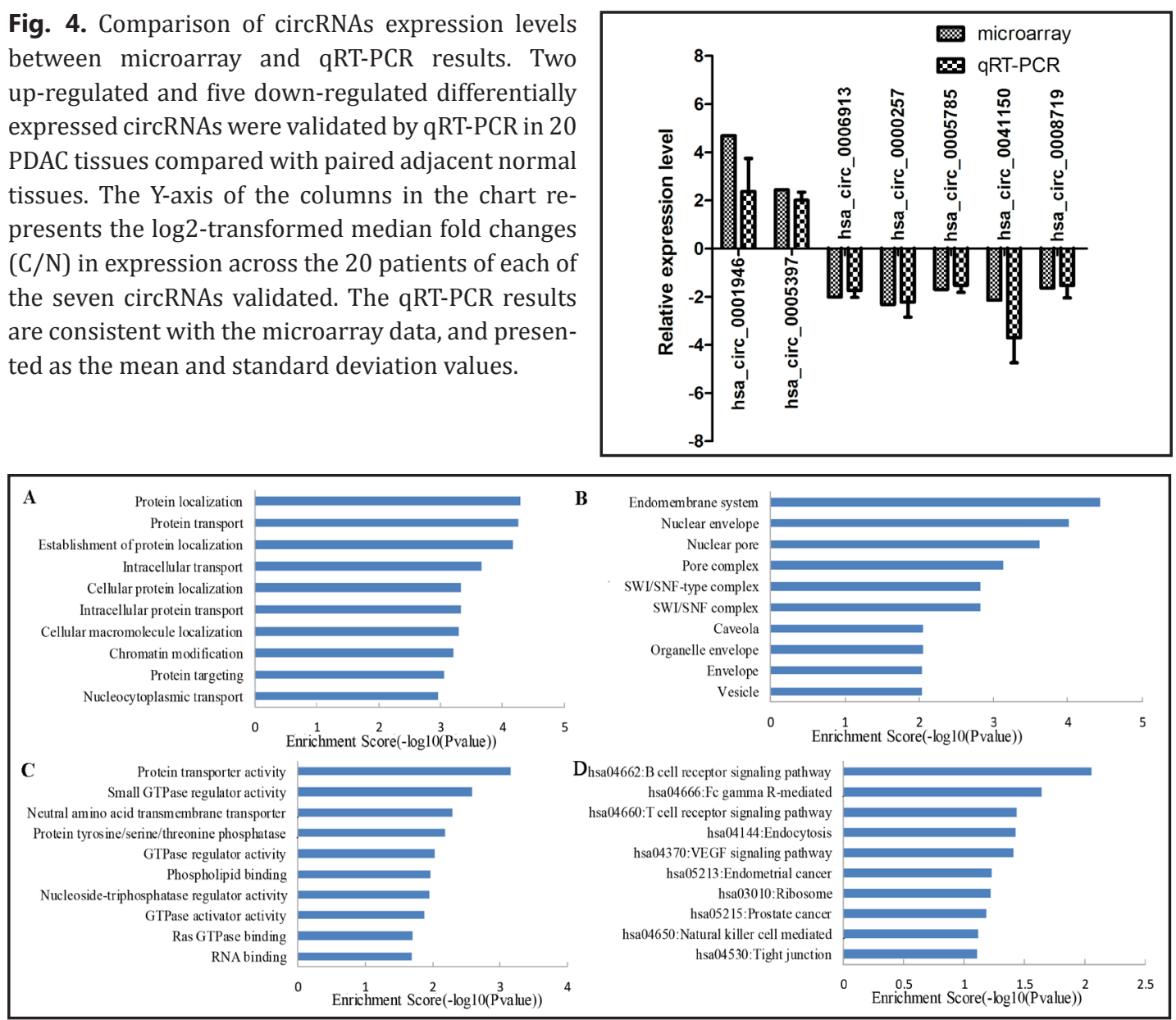

Fig. 5. GO enrichment and pathway analysis for dysregulated circRNAs gene symbols. (A) Most significantly enriched GO (- $\log 10$ ( $P$ value $)$ ) terms of circRNAs gene symbols according to biological process. (B) Most significantly enriched (- $\log 10$ ( $P$ value) $) \mathrm{GO}$ terms of circRNAs gene symbols according to cellular component. (C) Most significantly enriched $(-\log 10(P$ value $))$ GO terms of circRNAs gene symbols according to molecular function. (D) The bar plot shows the top ten enrichment score $(-\log 10$ ( $P$ value $)$ of the significantly enriched pathways.

significant enriched GO term in the biological process was protein localization (G0:0008104, $P=5.11 \mathrm{E}-05$ ) (Fig. 5A); the most significant enriched GO term in the cellular component was endomembrane system (GO:0012505, $P=3.68 \mathrm{E}-05$ ) (Fig. 5B); the most significant enriched GO term in the molecular function was protein transporter activity (G0:0008565, $P=6.94 \mathrm{E}$ 04) (Fig. 5C). The pathway analysis indicated that 10 pathways might be involved in the progression of PDAC (Fig. 5D). Among these pathways, the gene category "endocytosis, VEGF signaling pathway," has been reported to be involved in the progression of PDAC [27-30].

\section{Annotation for circRNA/microRNA Interaction}

Recent studies have reported that circRNAs could function as a miRNA sponge to regulate the gene expression $[11,19,31]$. CircRNAs can bind cancer-associated miRNAs to be involved in cancer-associated pathways [32-36]. To evaluate circRNAs potential functions, we investigated potential miRNAs binding with circRNAs. The circRNA/miRNA interaction was predicted using Arraystar's home-made miRNA target prediction software based on TargetScan and miRanda. Two confirmed circRNAs (hsa_circ_0005397 and hsa_ circ_0005785) were annotated in detail using the circRNA/miRNA interaction information (Fig. 6 ). 


\section{Discussion}

Pancreatic carcinogenesis is a complicated biological process that is characterized by a large number of molecular abnormalities. In recent decades, the molecular mechanism of PDAC has been extensively investigated. The gene and noncoding RNA, such as miRNA and IncRNA, expression microarrays have been recognized as a feasible and useful approach to profile the molecular signatures of PDAC $[37,38]$. Increasing studies are continuing to prove that noncoding RNAs, including miRNAs and IncRNAs, are involved in the development and procession of PDAC [38-40]. However, the tumorigenesis mechanisms of PDAC are far more elucidated. Interestingly, numerous circRNAs have recently been discovered. The circRNA acts as a new star of noncoding RNA, which expanded our knowledge in understanding the complexity of noncoding RNA. The circRNAs were originally thought to be by-products of splicing errors or the mRNA process [32]. Recent studies revealed that circRNAs are a stable, diverse and conserved class of RNA molecules $[11,12]$. Moreover, emerging evidence indicates that circRNAs may be involved in the procession of diseases, especially in cancer [32]. For example, circRNAs are globally reduced in colorectal cancer (CRC) tissues via analyses of RNA-sequencing data from 12 matched normal colon mucosa and tumor tissues [41]. The hsa_circ_002059 was first found to be significantly down-regulated in GC and may be a potential novel and stable biomarker for the diagnosis of GC [23]. The cir-ITCH expression is typically down-regulated in esophageal squamous cell carcinoma (ESCC) and CRC, and may have an inhibitory effect on ESCC and CRC by suppressing the Wnt/ $\beta$-catenin pathway $[19,42]$. Hence, these findings suggest that circRNAs play a crucial role in diseases, especially in cancer, and may serve as a new diagnosis and strategy for treating disease. However, to date, research regarding circRNAs dysregulation in PDAC has not been reported.

In this study, we investigated circRNAs expression profile of PDAC using the microarray analysis for the first time. We found that the circRNAs expression levels in PDAC samples were different from adjacent normal tissues. The microarray expression profiles exhibited that 209 up-regulated circRNAs and 142 down-regulated circRNAs were significantly differentially expressed ( $\mathrm{FC} \geq 1.5$ and $P<0.05$ ) in six paired PDAC samples. Moreover, we selected seven dysregulated circRNAs from the microarray data to examine the expression levels using qRT-PCR in 20 PDAC tissues and paired adjacent normal tissues. Our results showed that the qRT-PCR results and microarray data are consistent, indicating that the microarray data are reliable.

Otherwise, GO analysis and pathway analysis were analyzed to explore preliminarily the biological functions and potential mechanisms of circRNAs in the tumorigenesis of PDAC. GO enrichment analysis revealed that some genes symbol were involved in the regulation of biological process, celluar component and molecular function. Among the GO terms found in this study, SWI/SNF complex had been reported to play a important role in the development of PDAC [43]. Remarkably, among these pathways, "endocytosis and VEGF signaling pathway" have been reported to be involved in the progression of PDAC [27-30]. These findings indicated that several circRNAs might be involved in the development and progression of PDAC.

Recent studies have demonstrated that circRNAs could function as miRNA sponges or potent competitive endogenous RNA (ceRNA) molecules [31-35]. Given that miRNAs play important roles in the progression of PDAC [44], some circRNAs could likely be involved in PDAC via interacting with miRNAs. We found that the majority of circRNAs contained one or more miRNA binding sites based on the sequences analysis. The association of miRNAs with PDAC indicated that circRNAs may have a regulatory role in PDAC. For example, among the founded potential circRNA/miRNA interactions, hsa_circ_0005785 is potentially able to bind miR181a and miR181b. Moreover, miR-181a played a critical role in regulating pancreatic cancer growth and migration [45]. MiR-181b was associated with the resistance of pancreatic cancer cells to gemcitabine [46]. However, additional studies are required to clarify whether these circRNAs are involved in PDAC, and whether circRNAs acting as miRNA sponges are a general phenomenon. 
A hsa circ 0005397 vs hsa-miR-26b-3p

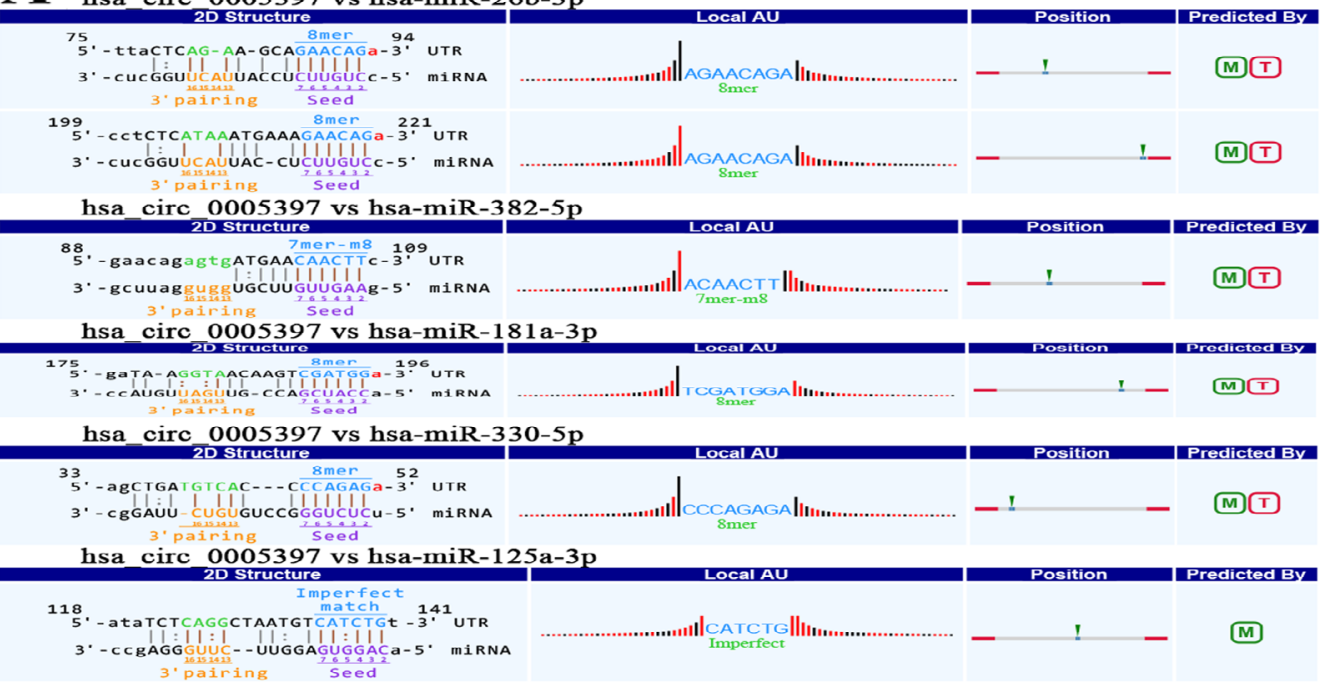

B hsa_circ_0005785 vs hsa-miR-181a-5p

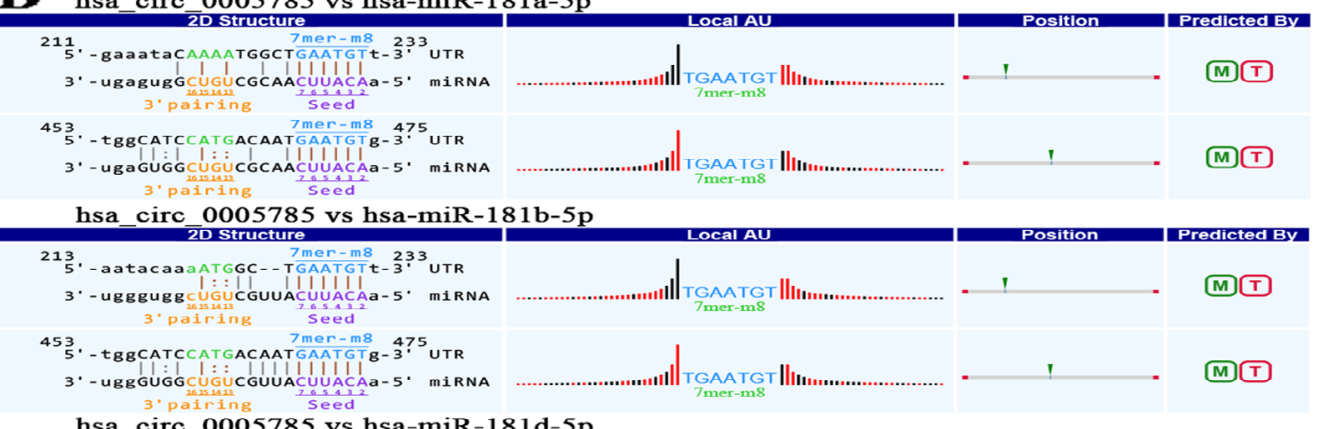

hsa_circ_0005785 vs hsa-miR-181d-5p
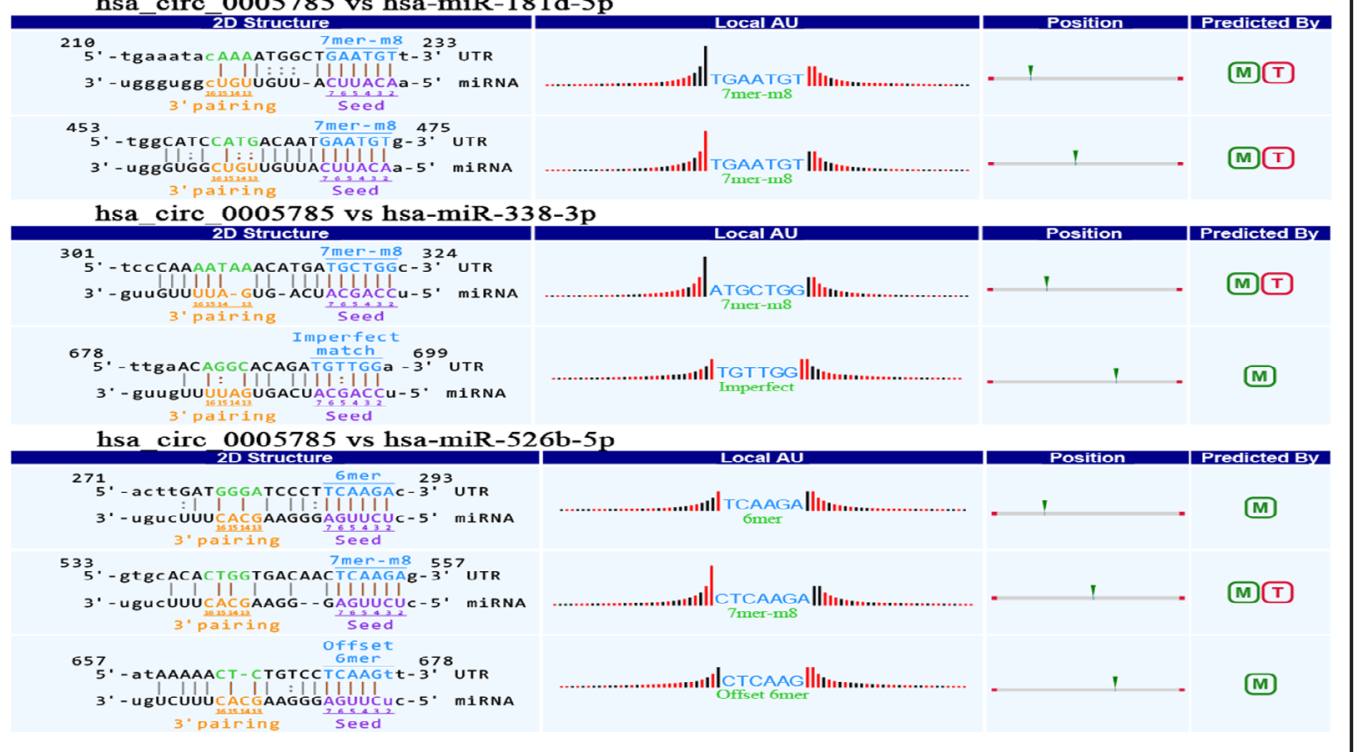

Fig. 6. A snippet of the detailed annotation for circRNA/miRNA interaction. (A) hsa_circ_0005397. (B) hsa_ circ_0005785. 8mer: bases from number 2 to 8 match perfectly, and number 1 base is A; 7mer-m8: bases from number 2 to 8 matchs perfectly, and number 1 base is not A; 6mer: bases from number 2 to 7 match perfectly, and number 1 base is not A; offset 6mer: bases from number 3 to 8 match perfectly; imperfect match: there is imperfect base match from number 2 to 7; M: circRNA/miRNA interaction can be predicted by miRanda; T: circRNA/miRNA interaction can be predicted by TargetScan. 


\section{Cellular Physiology Cell Physiol Biochem 2016;40:1334-1344

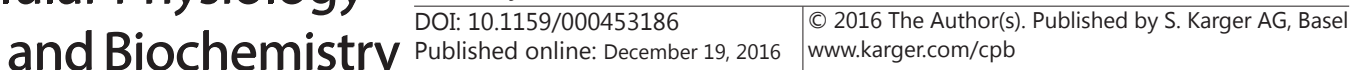 \\ Li et al.: Circular RNA Expression Profile in Pancreatic Cancer}

In conclusion, our study discovered that circRNAs are dysregulated in pancreatic ductal adenocarcinoma compared with adjacent normal tissues. For these dysregulated circRNAs, we conducted circRNAs gene symbols GO analysis and pathway analysis and predicted binding miRNAs. Such bioinformation would be used for investigating the functions of these circRNAs in the development and progression of PDAC. We also verify seven dysregulated circRNAs expression levels in 20 paired samples via qRT-PCR, which is consistent with the microarray data. Altogether, these findings provide potential targets for the future treatment of PDAC and novel insights into PDAC biology. Additional investigation of the circRNAs identified in this study will focus on their association with PDAC and biological functions.

\section{Acknowledgments}

We would like to thank Yan Qiu and Kun Liu (Department of Hepatobiliary Surgery of Xijing Hospital) for collecting the samples. We thank CapitalBio Corporation (Beijing, China) for his help in GO and pathway analysis. We are also grateful for the anonymous reviewers' constructive comments and suggestions. This work was supported by grants from the National Natural Science Foundation of China (No. 81672339) and the Health Public Welfare Industry Special Scientific Research Projects of China (No.201202007).

\section{Disclosure Statement}

The authors declare that they have no conflict of interest.

\section{References}

1 Ryan DP, Hong TS, Bardeesy N: Pancreatic adenocarcinoma. N Engl J Med 2014;371:2139-2041.

2 Hezel AF, Kimmelman AC, Stanger BZ, Bardeesy N, Depinho RA: Genetics and biology of pancreatic ductal adenocarcinoma. Genes Dev 2006;20:1218-1249.

3 Vincent A, Herman J, Schulick R, Hruban RH, Goggins M: Pancreatic cancer. Lancet 2011;378:607-620.

4 Hartwig W, Werner J, Jäger D, Debus J, Büchler MW: Improvement of surgical results for pancreatic cancer. Lancet Oncol 2013;14:e476-485.

5 Paulson AS, Tran Cao HS, Tempero MA, Lowy AM: Therapeutic advances in pancreatic cancer. Gastroenterology 2013;144:1316-1326.

6 Tay Y, Rinn J, Pandolfi PP: The multilayered complexity of ceRNA crosstalk and competition. Nature 2014;505:344-352.

7 Prensner JR, Chinnaiyan AM: The emergence of lncRNAs in cancer biology. Cancer Discov 2011;1:391-407.

8 Shi X, Sun M, Liu H, Yao Y, Song Y: Long non-coding RNAs: a new frontier in the study of human diseases. Cancer Lett 2013;339:159-166.

9 Chen LL, Yang L: Regulation of circRNA biogenesis. RNA Biol 2015;12:381-388.

10 Suzuki H, Tsukahara T: A view of pre-mRNA splicing from RNase R resistant RNAs. Int J Mol Sci 2014;15:9331-9342.

11 Memczak S, Jens M, Elefsinioti A, Torti F, Krueger J, Rybak A, Maier L, Mackowiak SD, Gregersen LH, Munschauer M, Loewer A, Ziebold U, Landthaler M, Kocks C, le Noble F, Rajewsky N: Circular RNAs are a large class of animal RNAs with regulatory potency. Nature 2013; 21;495:333-338.

12 Jeck WR, Sorrentino JA, Wang K, Slevin MK, Burd CE, Liu J, Marzluff WF, Sharpless NE: Circular RNAs are abundant, conserved, and associated with ALU repeats. RNA 2013;19:141-157.

13 Salzman J, Chen RE, Olsen MN, Wang PL, Brown PO: Cell-type specific features of circular RNA expression. PLoS Genet 2013;9:e1003777.

14 Rybak-Wolf A, Stottmeister C, Glažar P, Jens M, Pino N, Giusti S, Hanan M, Behm M, Bartok O, Ashwal-Fluss R, Herzog M, Schreyer L, Papavasileiou P, Ivanov A, Öhman M, Refojo D, Kadener S, Rajewsky N: Circular RNAs in the Mammalian Brain Are Highly Abundant, Conserved, and Dynamically Expressed. Mol Cell 2015;58:870-885. 


\section{Cellular Physiology Cell Physiol Biochem 2016;40:1334-1344 \begin{tabular}{l|l|l}
\hline DOI: 10.1159/000453186 & $\begin{array}{l}\text { () 2016 The Author(s). Published by S. Karger AG, Basel } \\
\text { www.karger.com/cpb }\end{array}$
\end{tabular} \\ Li et al.: Circular RNA Expression Profile in Pancreatic Cancer}

15 You X, Vlatkovic I, Babic A, Will T, Epstein I, Tushev G, Akbalik G, Wang M, Glock C, Quedenau C, Wang X, Hou J, Liu H, Sun W, Sambandan S, Chen T, Schuman EM, Chen W: Neural circular RNAs are derived from synaptic genes and regulated by development and plasticity. Nat Neurosci 2015;18:603-610.

16 Hansen TB, Kjems J, Damgaard CK: Circular RNA and miR-7 in cancer. Cancer Res 2013;73:5609-5612.

17 Boeckel JN, Jaé N, Heumüller AW, Chen W, Boon RA, Stellos K, Zeiher AM, John D, Uchida S, Dimmeler S: Identification and Characterization of Hypoxia-Regulated Endothelial Circular RNA. Circ Res 2015;117:884-890.

18 Satoh J, Yamamura T: Gene expression profile following stable expression of the cellular prion protein. Cell Mol Neurobiol 2004;24:793-814.

19 Li F, Zhang L, Li W, Deng J, Zheng J, An M, Lu J, Zhou Y: Circular RNA ITCH has inhibitory effect on ESCC by suppressing the Wnt/ $\beta$-catenin pathway. Oncotarget 2015;6:6001-6013.

20 Westholm JO, Miura P, Olson S, Shenker S, Joseph B, Sanfilippo P, Celniker SE, Graveley BR, Lai EC: Genomewide analysis of drosophila circular RNAs reveals their structural and sequence properties and agedependent neural accumulation. Cell Rep 2014;9:1966-1980.

21 Bahn JH, Zhang Q Li F, Chan TM, Lin X, Kim Y, Wong DT, Xiao X: The landscape of microRNA, Piwiinteracting RNA, and circular RNA in human saliva. Clin Chem 2015;61:221-230.

22 Burd CE, Jeck WR, Liu Y, Sanoff HK, Wang Z, Sharpless NE: Expression of linear and novel circular forms of an INK4/ARF-associated non-coding RNA correlates with atherosclerosis risk. PLoS Genet 2010;6:e1001233.

23 Li P, Chen S, Chen H, Mo X, Li T, Shao Y, Xiao B, Guo J: Using circular RNA as a novel type of biomarker in the screening of gastric cancer. Clin Chim Acta 2015;444:132-136.

24 Zhang Y, Zhang XO, Chen T, Xiang JF, Yin QF, Xing YH, Zhu S, Yang L, Chen LL: Circular intronic long noncoding RNAs. Mol Cell 2013;51:792-806.

25 Qu S, Song W, Yang X, Wang J, Zhang R, Zhang Z, Zhang H, Li H: Microarray expression profile of circular RNAs in human pancreatic ductal adenocarcinoma. Genom Data 2015;5:385-387.

26 Li Z, Huang C, Bao C, Chen L, Lin M, Wang X, Zhong G, Yu B, Hu W, Dai L, Zhu P, Chang Z, Wu Q, Zhao Y, Jia Y, Xu P, Liu H, Shan G: Exon-intron circular RNAs regulate transcription in the nucleus. Nat Struct Mol Biol 2015;22:256-264.

27 Marchand B, Arsenault D, Raymond-Fleury A, Boisvert FM, Boucher MJ: Glycogen synthase kinase-3 (GSK3) inhibition induces prosurvival autophagic signals in human pancreatic cancer cells. J Biol Chem 2015;290:5592-5605.

28 Yang A, Rajeshkumar NV, Wang X, Yabuuchi S, Alexander BM, Chu GC, Von Hoff DD, Maitra A, Kimmelman AC: Autophagy is critical for pancreatic tumor growth and progression in tumors with p53 alterations. Cancer Discov 2014;4:905-913.

29 Chen H, Zhang J, Luo J, Lai F, Wang Z, Tong H, Lu D, Bu H, Zhang R, Lin S: Antiangiogenic effects of

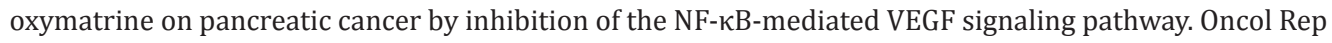
2013;30:589-595.

30 Sennino B, Ishiguro-Oonuma T, Wei Y, Naylor RM, Williamson CW, Bhagwandin V, Tabruyn SP, You WK, Chapman HA, Christensen JG, Aftab DT, McDonald DM: Suppression of tumor invasion and metastasis by concurrent inhibition of c-Met and VEGF signaling in pancreatic neuroendocrine tumors. Cancer Discov 2012;2:270-287.

31 Hansen TB, Jensen TI, Clausen BH, Bramsen JB, Finsen B, Damgaard CK, Kjems J: Natural RNA circles function as efficient microRNA sponges. Nature 2013;495:384-388.

32 Qu S, Yang X, Li X, Wang J, Gao Y, Shang R, Sun W, Dou K, Li H: Circular RNA: A new star of noncoding RNAs. Cancer Lett 2015;365:141-148.

33 Yang W, Du WW, Li X, Yee AJ, Yang BB: Foxo3 activity promoted by non-coding effects of circular RNA and Foxo3 pseudogene in the inhibition of tumor growth and angiogenesis. Oncogene 2016;35:3919-3931.

34 Wang K, Long B, Liu F, Wang JX, Liu CY, Zhao B, Zhou LY, Sun T, Wang M, Yu T, Gong Y, Liu J, Dong YH, Li N, Li PF: A circular RNA protects the heart from pathological hypertrophy and heart failure by targeting miR223. Eur Heart J 2016. pii: ehv713.

35 Zheng Q, Bao C, Guo W, Li S, Chen J, Chen B, Luo Y, Lyu D, Li Y, Shi G, Liang L, Gu J, He X, Huang S: Circular RNA profiling reveals an abundant circHIPK3 that regulates cell growth by sponging multiple miRNAs. Nat Commun 2016;7:11215. 


\section{Cellular Physiology Cell Physiol Biochem 2016;40:1334-1344 \begin{tabular}{ll|l} 
DOI: 10.1159/000453186 & $\begin{array}{l}\text { O 2016 The Author(s). Published by S. Karger AG, Basel } \\
\text { www.karger.com/cpb }\end{array}$
\end{tabular} \\ Li et al.: Circular RNA Expression Profile in Pancreatic Cancer}

36 Ghosal S, Das S, Sen R, Basak P, Chakrabarti J: Circ2Traits: a comprehensive database for circular RNA potentially associated with disease and traits. Front Genet 2013;4:283.

37 Bloomston M, Frankel WL, Petrocca F, Volinia S, Alder H, Hagan JP, Liu CG, Bhatt D, Taccioli C, Croce CM: MicroRNA expression patterns to differentiate pancreatic adenocarcinoma from normal pancreas and chronic pancreatitis. JAMA 2007;297:1901-1908.

38 Li J, Liu D, Hua R, Zhang J, Liu W, Huo Y, Cheng Y, Hong J, Sun Y: Long non-coding RNAs expressed in pancreatic ductal adenocarcinoma and IncRNA BC008363 an independent prognostic factor in PDAC. Pancreatology 2014;14:385-390.

39 Dang Z, Xu WH, Lu P, Wu N, Liu J, Ruan B, Zhou L, Song WJ, Dou KF: MicroRNA-135a inhibits cell proliferation by targeting Bmi1 in pancreatic ductal adenocarcinoma. Int J Biol Sci 2014;10:733-745.

40 Pang EJ, Yang R, Fu XB, Liu YF: Overexpression of long non-coding RNA MALAT1 is correlated with clinical progression and unfavorable prognosis in pancreatic cancer. Tumour Biol 2015;36:2403-2407.

41 Bachmayr-Heyda A, Reiner AT, Auer K, Sukhbaatar N, Aust S, Bachleitner-Hofmann T, Mesteri I, Grunt TW, Zeillinger R, Pils D: Correlation of circular RNA abundance with proliferation--exemplified with colorectal and ovarian cancer, idiopathic lung fibrosis, and normal human tissues. Sci Rep 2015;5:8057.

42 Huang G, Zhu H, Shi Y, Wu W, Cai H, Chen X: cir-ITCH plays an inhibitory role in colorectal cancer by regulating the Wnt/ $\beta$-catenin pathway. PLoS One 2015;10:e0131225.

43 Shain AH, Giacomini CP, Matsukuma K, Karikari CA, Bashyam MD, Hidalgo M, Maitra A, Pollack JR: Convergent structural alterations define SWItch/Sucrose NonFermentable (SWI/SNF) chromatin remodeler as a central tumor suppressive complex in pancreatic cancer. Proc Natl Acad Sci U S A 2012;109:E252-259.

44 Song B, Zheng K, Ma H, Liu A, Jing W, Shao C, Li G, Jin G: miR-429 determines poor outcome and inhibits pancreatic ductal adenocarcinoma growth by targeting TBK1. Cell Physiol Biochem 2015;35:1846-1856.

45 Zhang P, Guo Z, Hu R, He X, Jiao X, Zhu X: Interaction between microRNA-181a and TNFAIP1 regulates pancreatic cancer proliferation and migration. Tumour Biol 2015;36:9693-9701.

46 Takiuchi D, Eguchi H, Nagano H, Iwagami Y, Tomimaru Y, Wada H, Kawamoto K, Kobayashi S, Marubashi S, Tanemura M, Mori M, Doki Y: Involvement of microRNA-181b in the gemcitabine resistance of pancreatic cancer cells. Pancreatology 2013;13:517-523. 